\title{
Consumo de alcohol y factores asociados en futbolistas cadetes españoles (14-16 años) Alcohol consumption and associated factors in Spanish football players from the cadet category (14-16 years) \\ *Olegario Jesús González-Ramos, **Magdalena Marrero-Montelongo, Carmen Navarro-Rodríguez, Milagros Torres-García *Consejería de Educación. Gobierno de Canarias (España), ** Universidad de Las Palmas de Gran Canaria (España)
}

Resumen. El deporte en la adolescencia se asocia a un estilo de vida saludable, asignándole diversos autores un efecto protector ante el consumo de alcohol. El objetivo del trabajo fue identificar el consumo de alcohol y los factores asociados a éste, en los futbolistas de la categoría cadete de la provincia de Las Palmas. Estudio descriptivo transversal en el que se estudió a 308 jugadores, determinados mediante muestreo por clúster, seleccionando una muestra aleatoria simple de 20 equipos. El instrumento de recogida de datos fue un cuestionario ad hoc. Se realizó un análisis descriptivo de los datos (SPSS 17.0 para Windows), empleándose para la comparación de proporciones el test de la X². El 56.2\% de los jugadores reconoció beber actualmente. El 13.6\% declaró haberlo hecho al menos cuatro días en los últimos 30 días, el 32.2\% haberse emborrachado en los últimos 12 meses y el $39.9 \%$ haber ingerido tres o más vasos seguidos en los últimos 12 meses, práctica ésta última significativamente mayor entre los que jugaban en preferente ( $p<.001$ ). El consumo de alcohol se asoció a diversos factores externos a la práctica deportiva, entre los que figuraban, la práctica de otras conductas de riesgo para la salud $(p<.01)$, la percepción de no servir para la escuela $(p<.001)$, o la percepción de un estilo educativo parental poco controlador y carente de afecto $(p<.05)$. La notable proporción de consumidores de alcohol entre los jugadores pone de manifiesto, la necesidad de estrategias de intervención para su prevención a través de los clubes de fútbol.

Palabras claves: alcohol; fútbol; adolescentes; drogas; violencia.

Abstract. Many authors consider sport among adolescents to have a protective effect against alcohol consumption. The former is in fact associated to a healthy lifestyle. The aim of this research was to identify alcohol consumption and factors linked to it among football players from the cadet category in the province of Las Palmas, Spain. The present work adopted a cross-sectional descriptive analysis of 308 players selected based on cluster sampling from a simple random sample of 20 teams. The data collection instrument was an ad hoc questionnaire. A descriptive data analysis was conducted (SPSS 17.0 for Windows), and the $\mathrm{X}^{2}$ test was applied for proportions comparison. 56.2\% of the football players studied admitted consuming alcohol. $13.6 \%$ stated to have drunk at least during four of the last 30 days, $32.2 \%$ declared to have been drunk in the last 12 months, and $29.9 \%$ said to have drunk consecutively three or more glasses in the last 12 months. This last practice was significantly greater among those players belonging to the higher division $(p<.001)$. Alcohol consumption was linked to different factors unrelated to the practice of sport, including engaging in other health risk behaviours $(p<.01)$, having low academic self-esteem $(p<.001)$, or perceiving parents to exert little control over them and to be unaffectionate $(p<.05)$. The significant proportion of alcohol consumers among football players highlights the need to implement preventive intervention strategies in football clubs.

Key words: alcohol; football; adolescents; drugs; violence.

\section{Introducción}

El consumo de riesgo de alcohol, término utilizado por la Organización Mundial de la Salud, es definido por la misma como aquel nivel o patrón de consumo que produce efectos nocivos para la salud, físicos, mentales y sociales. A diferencia del perjudicial, el consumo de riesgo hace referencia a modelos de consumo que tienen importancia para la salud pública, aunque el individuo consumidor no presente ninguna alteración en ese momento (OMS, 2008). Los criterios de consumo de riesgo varían en función de diferentes aspectos, entre los que figura la edad de los bebedores.

En la adolescencia, período de la vida en el que la persona tiene derecho a desarrollarse en un entorno protegido de las consecuencias negativas del consumo de alcohol, la práctica de un consumo de riesgo es un hecho que preocupa y ha de ocupar cada vez más a los responsables de la salud pública, dados los efectos a corto, medio y largo plazo que dicha conducta puede ocasionar en una población en proceso de maduración física, psíquica y social (García-Moreno, Expósito, Sanhueza \& Angulo, 2008; Motos, Cortés, Giménez \& Cadaveira, 2015; MoureRodríguez, Doallo, Juan-Salvadores, Corral, Cadaveira \& CaamañoIsorna, 2016).

La importancia del consumo de alcohol de riesgo entre los adolescentes españoles se ve reflejada en los datos de frecuencia presentados en ESTUDES 2014/15 (Ministerio de Sanidad, Servicios Sociales e Igualdad, 2016), que muestran que en los últimos 30 días el 22.2\% de los escolares de 14-18 años se ha emborrachado y el 32.2\% ha tomado cinco o más vasos, cañas o copas de bebidas alcohólicas en una misma ocasión, siendo esta última conducta algo superior entre los varones. Datos similares al respecto, aporta el estudio Health Behaviour in SchoolAged Children (HBSC)-2014 (Moreno, et al., 2016), llevado a cabo también en estudiantes de nuestro país, de 11-18 años.

Consecuentemente, urge la identificación de factores que protejan a los adolescentes de la adopción de estas conductas de riesgo, y cuya promoción contribuya a disminuir en la población de esta edad la incidencia y prevalencia de estas prácticas de riesgo. La búsqueda de factores protectores es especialmente necesaria en países como el nuestro, en los que el consumo de bebidas alcohólicas se encuentra consolidado en sus costumbres sociales (Alfonso, Huedo-Medina \& Espada, 2009; Vaquero-Cristóbal, Isorna \& Ruiz, 2012) aceptándose, incluso, la incorporación de los adolescentes a dicho consumo como una forma más de relación social. Esta consideración del consumo de bebidas alcohólicas como acto social, cultural o gastronómico, favorece el contacto de los adolescentes con el mismo.

El deporte, admitido como un comportamiento integrante de un estilo de vida saludable al que se le ha atribuido un carácter protector frente al consumo de sustancias tóxicas (López, Rodríguez, García \& Pérez, 2016; Sañudo, de Hoyo \& Corral, 2009), ha sido uno de los factores más postulados como protector del consumo de alcohol en la adolescencia. Sin embargo, hasta el momento, las diferencias existentes entre los datos aportados por los estudios realizados en este sentido no permiten atribuirle dicho papel de manera consistente, ni justificar la priorización de su inclusión en los programas dirigidos a prevenir este tipo de comportamiento en la adolescencia, requiriéndose más investigación en esta línea (Lema, Salazar, Varela, Tamayo, Rubio \& Botero, 2009; VanKim, Nelson, Ehlinger, Lust \& Story, 2010).

Por todo ello, el objetivo de este trabajo fue identificar el consumo de alcohol y los factores asociados a éste, en jugadores de futbol federados pertenecientes a los 147 equipos de la categoría cadete de primer y segundo año, de la provincia de Las Palmas, con edades comprendidas entre los 14 y los 16 años.

\section{Método}

\section{Diseñoy participantes}

Estudio descriptivo transversal cuya población de referencia fue- 
ron los 2.205 jugadores federados pertenecientes a los 147 equipos de la categoría cadete de primer y segundo año, de la provincia de Las Palmas, con edades comprendidas entre los 14 y los 16 años. Para la determinación del tamaño muestral se realizó un muestreo por clúster, seleccionando una muestra aleatoria simple de 20 equipos (308 jugadores). Se tuvo en cuenta la prevalencia de consumo de alcohol obtenida en un estudio piloto previamente realizado en la zona norte de Gran Canaria en el que se encuestaron a los jugadores de 8 equipos, lo que permitió estimar la tasa de bebedores con una cota de error inferior al 5\%.

\section{Instrumento de recogida de datos}

Los datos han sido recogidos a través de un cuestionario ad hoc diseñado para ser cumplimentado por los jugadores y para cuya realización se revisaron previamente los cuestionarios validados del estudio Health Behaviour in School-aged Children (HBSC)-2010 (Moreno, et al., 2012), y de la Encuesta estatal sobre uso de drogas en estudiantes de enseñanzas secundarias ESTUDES 2010 (Ministerio de Sanidad, Política Social e Igualdad, 2012). Se llevó a cabo un proceso de selección y adaptación de las preguntas y se diseñó una primera versión del cuestionario. Posteriormente, fue pilotado en adolescentes de edades y características similares a los de nuestro estudio, quedando constituido el cuestionario definitivo por 6 preguntas sobre datos socioeconómicos, 37 preguntas derivadas del HBSC relacionadas con los hábitos de vida y 9 preguntas procedentes del ESTUDES relacionadas con el patrón de consumo de alcohol.

\section{Variables}

Las variables objeto de estudio se muestran en la tabla 1. El consumo experimental de bebidas alcohólicas, el consumo actual, y el consumo de riesgo se analizaron en relación a cinco grupos de factores: la división en la que jugaban, sus propias conductas relacionadas con la salud, las percepciones que tenían sobre sus habilidades personales y sus conocimientos sobre el alcohol, las características percibidas sobre su entorno familiar, y las percepciones sobre conductas desarrolladas por el entorno de amigos.

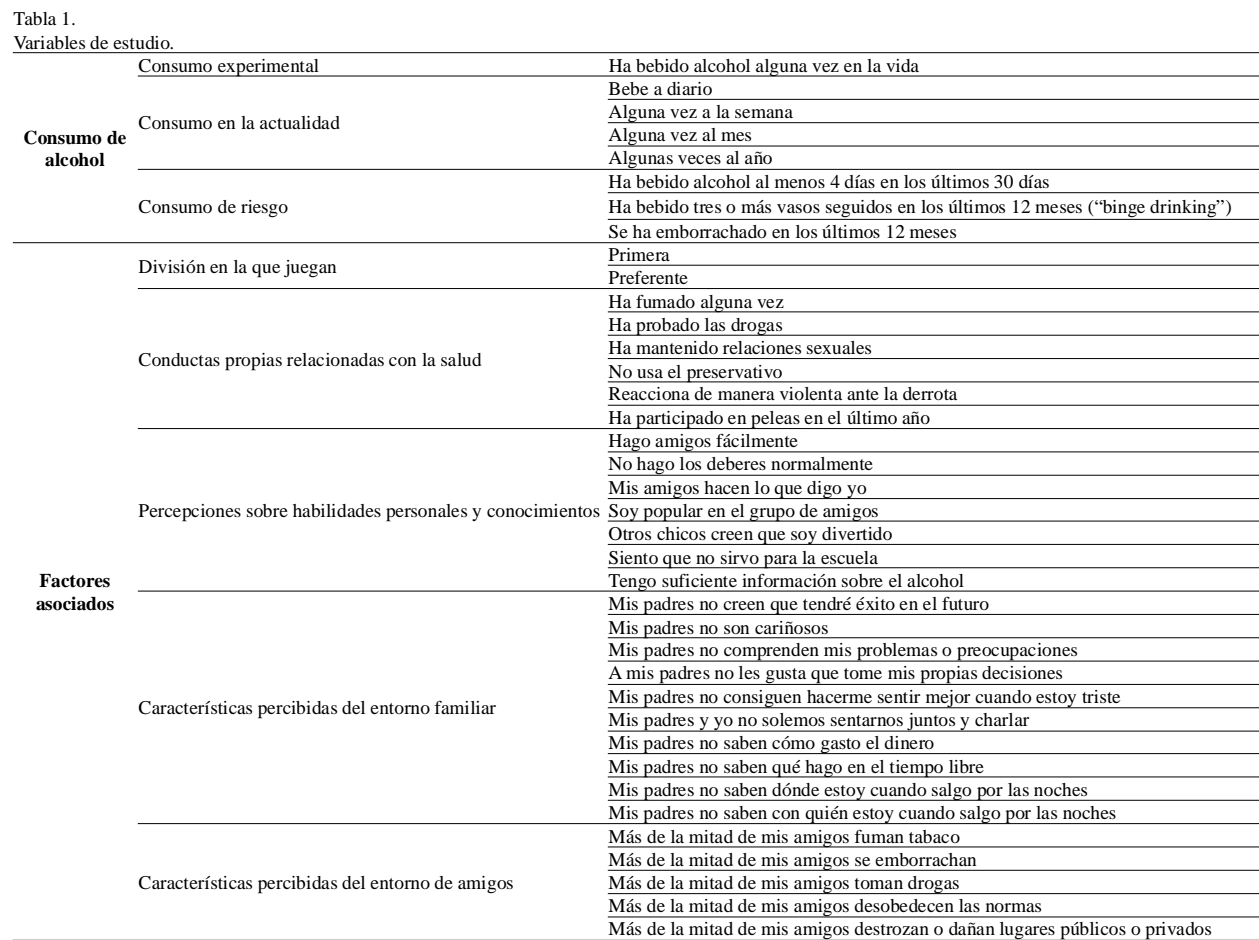
abril de 2014

\section{Análisis estadístico} inferior a 0.05

\section{Resultados}

los equipos seleccionados en los momentos previos al entrenamiento, informándoles del carácter anónimo del mismo. Dicho cuestionario fue cumplimentado por los futbolistas en presencia del investigador. Esto tuvo lugar durante el período comprendido entre los meses de febrero y

El análisis de los datos se llevó a cabo a través del paquete estadístico del programa SPSS 17.0 para Windows. Para las variables categóricas, se llevó a cabo la distribución de frecuencias. Para las variables cuantitativas, se calcularon medidas de tendencia central (media) y medidas de dispersión (desviación típica). Para estudiar la posible asociación entre variables se realizó la prueba de la Ji-Dos, partiendo siempre de la independencia de las mismas. Un test de hipótesis se consideró estadísticamente significativo, cuando el correspondiente p valor fue

\begin{tabular}{|c|c|}
\hline Variables & n (\%) \\
\hline \multicolumn{2}{|l|}{ División en la que jugaban } \\
\hline Primera & $185(60.1)$ \\
\hline Preferente & $123(39.9)$ \\
\hline \multicolumn{2}{|l|}{ Grupo categoría cadete } \\
\hline Primer año & $98(31.8)$ \\
\hline Segundo año & $210(68.2)$ \\
\hline \multicolumn{2}{|l|}{ Estudios que cursaban } \\
\hline Educación Secundaria Obligatoria (ESO) & $306(99.4)$ \\
\hline Programas de Cualificación Profesional Inicial (PCPI) & $2(.6)$ \\
\hline \multicolumn{2}{|l|}{ Motivos para la práctica de actividad física } \\
\hline Motivos extrínsecos (gusto, diversión, encuentro con amigos) & $246(81.2)$ \\
\hline Motivos intrínsceos (salud, forma física, competición) & $57(18.8)$ \\
\hline Edad (años) (media \pm DT) & $14.69 \pm 0.46$ \\
\hline Peso (kilogramos) (media $\pm D T)$ & $64.07 \pm 10.36$ \\
\hline Talla (metros) (media \pm DT) & $1.72 \pm 0.07$ \\
\hline IMC (media \pm DT) & $21.47 \pm 3.07$ \\
\hline Días a la semana que dedicaban a practicar fútbol (media \pm DT) & $3.77 \pm 0.61$ \\
\hline Horas de entrenamiento diario (media \pm DT) & $1.66 \pm 0.35$ \\
\hline
\end{tabular}

La población estudiada quedó constituida por la totalidad de los integrantes de los 20 equipos de fútbol de la categoría cadete de la provincia de Las Palmas seleccionados en el muestreo, lo que supuso un total de 308 jugadores. En la tabla 2 se muestran las características generales de la misma.

\section{Prevalencia del consu- mo de bebidas alcohólicas}

$\mathrm{Al}$ analizar la prevalencia de consumo de bebidas alcohólicas, el $60.1 \%$ de los futbolistas declaró haberlas consumido alguna vez en su vida y el 56.2\% reconoció hacerlo en la actualidad, siendo el patrón de consumo más frecuentemente seguido el de alguna vez al mes (24\%).

La distribución de la población según las variables de consumo de riesgo se muestra asimismo en la tabla 3.

$\mathrm{Al}$ analizar la prevalencia del consumo experimental, actual y de riesgo de bebidas alcohólicas, según la cate-

\section{Procedimiento}

Una vez obtenida la muestra, se contactó con los directivos y cuerpos técnicos de cada uno de los 20 equipos seleccionados para explicarles los objetivos del estudio y solicitar su colaboración y consentimiento. Posteriormente se pasó el cuestionario a los integrantes de goría en la que jugaban los futbolistas cadetes, se observó que tanto el consumo experimental, como el actual, así como el consumo de tres o más vasos seguidos en los últimos 30 días, fueron más frecuentes entre los futbolistas que jugaban en la división preferente que entre los que lo hacían en primera $(p<.05)$. 
Tabla 3

Consumo experimental, actual y de riesgo de bebidas alcohólicas según división en donde juegan.

\begin{tabular}{|c|c|c|c|c|}
\hline \multirow[t]{2}{*}{ Variables } & Total & $\begin{array}{l}\text { Primera } \\
(\mathrm{N}=185)\end{array}$ & $\begin{array}{c}\text { Preferente } \\
(\mathrm{N}=123)\end{array}$ & \multirow[t]{2}{*}{$\begin{array}{r}p- \\
\text { valor }\end{array}$} \\
\hline & n (\%) & n (\%) & $\mathrm{n}(\%)$ & \\
\hline \multicolumn{5}{|l|}{ Consumo experimental de alcohol ( $\mathrm{N}=308)$} \\
\hline Ha bebido alcohol alguna vez en la vida & $185(60.1)$ & $102(55.1)$ & $83(67.5)$ & $.030^{*}$ \\
\hline No ha bebido alcohol & $123(39.9)$ & $83(44.9)$ & $40(32.5)$ & \\
\hline $\begin{array}{l}\text { Edad inicio consumo de alcohol (N=185) (años) } \\
(\text { media } \pm D T)\end{array}$ & \multicolumn{4}{|c|}{$13.46 \pm 1.18$} \\
\hline \multicolumn{5}{|l|}{ Consumo de alcohol en la actualidad ( $\mathrm{N}=308$ ) } \\
\hline No bebe alcohol en la actualidad & $135(43.8)$ & $91(49.2)$ & $44(35.8)$ & \\
\hline Bebe a diario & $2(0.6)$ & $1(0.5)$ & $1(0.8)$ & \\
\hline Alguna vez a la semana & $26(8.4)$ & $11(5.9)$ & $15(12.2)$ & \\
\hline Alguna vez al mes & $74(24)$ & $41(22.2)$ & $33(26.8)$ & $.020^{*}$ \\
\hline Algunas veces al año & $71(23.1)$ & $41(22.2)$ & $30(24.4)$ & \\
\hline \multicolumn{5}{|l|}{ Consumo de riesgo de alcohol. } \\
\hline $\begin{array}{l}\text { Ha bebido alcohol al menos } 4 \text { días en los últimos } 30 \\
\text { días ( } N=308 \text { ) }\end{array}$ & $42(13.6)$ & $21(11.4)$ & $21(16.8)$ & .152 \\
\hline $\begin{array}{l}\text { dlas }(N=308) \\
\text { Ha bebido tres o más vasos seguidos en los últimos } 12\end{array}$ & $123(39.9)$ & $59(31.9)$ & $64(52)$ & $.001^{*}$ \\
\hline $\begin{array}{l}\text { meses (“binge drinking”) (N=308) } \\
\text { Se ha emborrachado en los últimos } 12 \text { meses (N=308) }\end{array}$ & $99(32.2)$ & $53(28.6)$ & $46(37.4)$ & 107 \\
\hline Edad "binge drinking” ( $\mathrm{N}=123)$ (años) (media $\pm \mathrm{DT})$ & \multicolumn{4}{|c|}{$13.79 \pm 1.3$} \\
\hline Edad primera borrachera $(\mathrm{N}=35)$ (años) (media $\pm \mathrm{DT})$ & \multicolumn{4}{|c|}{$14.14 \pm .97$} \\
\hline
\end{tabular}

futbolistas declaró haber participado en peleas en el último año y el $10.1 \%$ reaccionar violentamente ante la derrota, siendo estas conductas, las prácticas sexuales y el consumo de otras sustancias tóxicas más frecuentes entre los jugadores consumidores de alcohol $(p<.05)$.

En relación con las habilidades personales percibidas, se encontró que el 63.6\% de los deportistas se consideraba popular en el grupo de amigos, y el 67.2\% que los amigos hacían lo que él decía, siendo ambas percepciones más frecuentes entre los consumidores de alcohol $(p<.05)$, Asimismo se observó asociación estadísticamente significativa entre ser consumidor de bebidas alcohólicas en la actualidad y la percepción de no servir para la escuela y no hacer los deberes normalmente $(p<.05)$. El $80.2 \%$ de los futbolistas declaró tener suficiente información sobre el alcohol, siendo más frecuente entre los consumidores de alcohol $(p<.05)$.

En cuanto a las características percibidas del entorno familiar, que se muestran también en la tabla 4, se encontró que entre los futbolistas consumidores de alcohol fueron más frecuentes las percepciones analizadas ( $p<.05)$, exceptuando la relacionada con que los padres no saben con quién están cuando salen por las noches, para la que no se encontró diferencia estadísticamente significativa.

Un entorno de iguales caracterizado por amigos que fuman, se emborrachan, consumen drogas, desobedecen las normas o destrozan lugares públicos, fue también percibido de manera más frecuente por los futbolistas cadetes consumidores de alcohol $(p<.05)$.

Factores asociados al consumo de riesgo de bebidas alcohólicas

En la tabla 5, se muestran los resultados que relacionan de manera estadísticamente significativa las variables que caracterizan el consumo de riesgo de bebidas alcohólicas con otras conductas relacionadas con la salud, desarrolladas por los futbolistas, sus habilidades personales, las características
Factores asociados al consumo actual de bebidas alcohólicas

Los datos del análisis del consumo de bebidas alcohólicas en la actualidad por parte de los futbolistas cadetes en función de sus propias conductas relacionadas con la salud, las percepciones que tenían sobre sus habilidades personales y sus conocimientos sobre el alcohol, las características percibidas sobre su entorno familiar, y las percepciones sobre conductas desarrolladas por el entorno de amigos, se muestran en la tabla 4.

Cabe destacar entre todas las conductas analizadas, que el $48.7 \%$ de los
Tabla 5.

Conductas relacionadas con la salud y consumo de riesgo.

\begin{tabular}{|c|c|c|c|c|c|c|c|c|c|}
\hline \multirow{3}{*}{ Variables } & \multicolumn{3}{|c|}{$\begin{array}{l}\text { Beber alcohol al menos } 4 \text { días } \\
\text { en los últimos } 30 \text { días }\end{array}$} & \multicolumn{3}{|c|}{$\begin{array}{l}\text { Beber } 3 \text { ó más vasos seguidos } \\
\text { en los últimos } 12 \text { meses }\end{array}$} & \multicolumn{3}{|c|}{$\begin{array}{l}\text { Emborracharse en los } \\
\text { últimos } 12 \text { meses }\end{array}$} \\
\hline & $\mathrm{Si}$ & No & & $\mathrm{Si}$ & No & & $\mathrm{Si}$ & No & \\
\hline & n (\%) & n (\%) & & n (\%) & n (\%) & & n(\%) & n (\%) & valor \\
\hline \multicolumn{10}{|l|}{ Conductas propias relacionadas con la salud } \\
\hline Ha fumado alguna vez & $13(31)$ & $21(7.9)$ & $.001 *$ & $28(22.8)$ & $6(3.2)$ & $.001 *$ & $24(24.2)$ & $10(4.8)$ & $.001^{*}$ \\
\hline Bebe alcohol actualmente & $42(100)$ & $131(49.2)$ & $.001 *$ & $112(91.1)$ & $61(33)$ & $.001 *$ & $91(91.9)$ & $82(39.2)$ & $.001^{*}$ \\
\hline Ha probado las drogas & $12(28.6)$ & $21(7.9)$ & $.001 *$ & $29(23.6)$ & $4(2.2)$ & $.001 *$ & $25(25.3)$ & $8(3.8)$ & $.001 *$ \\
\hline Ha mantenido relaciones sexuales & $28(66.7)$ & $89(33.5)$ & $.001 *$ & $67(54.5)$ & $50(27)$ & $.001 *$ & 56 (56.6) & $61(29.2)$ & $.001^{*}$ \\
\hline No usa el preservativo & $9(32.1)$ & $11(12.4)$ & $.015^{*}$ & $14(20.9)$ & $6(12)$ & NS & $15(26.8)$ & $5(8.2)$ & $.008^{*}$ \\
\hline Reacciona de manera violenta ante la derrota & $9(21.4)$ & $22(8.3)$ & $.008 *$ & $21(17.1)$ & $10(5.4)$ & $.001 *$ & $16(16.2)$ & $15(7.2)$ & $.014 *$ \\
\hline Ha participado en peleas en el último año & $32(76.2)$ & $118(44.4)$ & $.001 *$ & $74(60.2)$ & $76(41.1)$ & $.001^{*}$ & $63(63.6)$ & $87(41.6)$ & $.001 *$ \\
\hline \multicolumn{10}{|l|}{$\begin{array}{l}\text { Percepciones sobre habilidades personales y conocimientos } \\
\text { s. }\end{array}$} \\
\hline Hago amigos fácilmente & $27(64.3)$ & $128(48.1)$ & $.050^{*}$ & $73(59.3)$ & $82(44.3)$ & $.010^{*}$ & $61(61.6)$ & $94(45)$ & $.006^{*}$ \\
\hline No hago los deberes normalmente & $31(73.8)$ & $168(63.2)$ & NS & $95(77.2)$ & $104(56.2)$ & $.001 *$ & 78 (78.8) & $121(57.9)$ & $.001 *$ \\
\hline Mis amigos hacen lo que digo yo & $37(88.1)$ & 170 (63.9) & $.002 *$ & $91(74)$ & $116(62.7)$ & $.039 *$ & 76 (76.8) & $131(62.7)$ & $.014 *$ \\
\hline Otros chicos creen que soy divertido & $37(88.1)$ & $234(88)$ & NS & $114(92.7)$ & 157 (84.9) & $.039 *$ & $93(93.9)$ & $178(85.2)$ & $.027 *$ \\
\hline Tengo suficiente información sobre el alcohol & $40(95.2)$ & $207(77.8)$ & $.008^{*}$ & $106(86.2)$ & $141(76.2)$ & $.032 *$ & $84(84.8)$ & $163(78)$ & NS \\
\hline \multicolumn{10}{|l|}{ Características percibidas del entorno familiar } \\
\hline Mis padres no saben cómo gasto el dinero & $21(50)$ & $67(25.2)$ & $.001 *$ & $46(37.4)$ & $42(22.7)$ & $.005 *$ & $42(42.4)$ & $46(22)$ & $.001^{*}$ \\
\hline Mis padres no saben dónde estoy cuando salgo por las noches & $17(40.5)$ & $42(15.8)$ & $.001 *$ & $36(29.3)$ & $23(12.4)$ & $.001 *$ & $35(35.4)$ & $24(11.5)$ & $.001 *$ \\
\hline Mis padres no saben con quién estoy cuando salgo por noches & $15(35.7)$ & $45(16.9)$ & $.004 *$ & $31(25.2)$ & $29(15.7)$ & $.039 *$ & $28(28.3)$ & $32(15.3)$ & $.007^{*}$ \\
\hline \multicolumn{10}{|l|}{ Características percibidas del entorno de amigos } \\
\hline Mis amigos fuman tabaco $* *$ & $9(25.7)$ & $22(9.2)$ & $.004 *$ & $22(20.4)$ & $9(5.5)$ & $.001 *$ & $19(21.3)$ & $12(6.5)$ & $.001^{*}$ \\
\hline Mis amigos se emborrachan $* *$ & $33(84.6)$ & $119(49.8)$ & $.001 *$ & $86(76.1)$ & $66(40)$ & $.001 *$ & 74 (79.6) & $78(42.2)$ & $.001^{*}$ \\
\hline Mis amigos toman drogas ** & $11(31.4)$ & $15(6.6)$ & $.001 *$ & $19(17.9)$ & $7(4.5)$ & $.001 *$ & $15(17.9)$ & $11(6.1)$ & $.003^{*}$ \\
\hline Mis amigos desobedecen las normas $* *$ & $22(64.7)$ & $65(32.3)$ & $.001 *$ & $45(46.9)$ & $42(30.2)$ & $.009 *$ & $38(49.4)$ & $49(31)$ & $.006 *$ \\
\hline Mis amigos destrozan o dañan lugares ** & $10(28.6)$ & $42(19.4)$ & NS & $30(28)$ & $22(15.3)$ & $.014^{*}$ & $27(31)$ & $25(15.2)$ & $.003^{*}$ \\
\hline
\end{tabular}


percibidas de su entorno familiar y de su grupo de amigos.

Se observó que la adopción de conductas como fumar, beber alcohol, probar drogas, mantener relaciones sexuales, reaccionar violentamente ante las derrotas y participar en peleas en el último año eran más frecuentes entre aquellos futbolistas que desarrollaban un consumo de alcohol de riesgo $(p<.05)$, siendo también más prevalentes en esta población, las percepciones de hacer amigos fácilmente y de liderazgo entre los iguales $(p<.05)$.

El consumo de riesgo de bebidas alcohólicas, se asoció de manera estadísticamente significativa $(p<.05)$ a un entorno familiar caracterizado por padres que no saben cómo gasta el dinero su hijo, dónde está su hijo, ni con quién está cuando sale por las noches.

En relación al entorno de amigos, la percepción de tener amigos que fuman, se emborrachan, toman drogas y desobedecen las normas fue también más frecuente entre aquellos deportistas que consumían alcohol de manera abusiva $(p<.05)$.

\section{Discusión}

La práctica de actividad física y deportiva constituye una conducta propia de un estilo de vida saludable, asignándole muchos autores un efecto protector ante el consumo de drogas (Castillo, Balaguer \& GarcíaMerita, 2007; Lázaro, 2011; López, et al., 2016; Meseguer, 2008; Moore \& Werch, 2005; Sañudo, et al., 2009; Vaquero-Cristóbal, et al., 2012). En otras investigaciones no obstante (Lorente, Souville, Griffet \& Grélot, 2004; Peck, Vida \& Eccles, 2008; Poortinga, 2007; Veliz, Boyd \& McCabe, 2015), se ha puesto de manifiesto un elevado consumo de dichas sustancias de abuso entre deportistas.

Los resultados de nuestro estudio muestran una prevalencia de consumo experimental de alcohol, entendido como haber tomado alguna bebida alcohólica alguna vez en la vida, del $60.1 \%$, dato inferior a los encontrados por otros autores en adolescentes españoles (Espada, Méndez \& Hidalgo, 2000; Ministerio de Sanidad, Servicios Sociales e Igualdad, 2016; López, Antolín, Barceló, Pérez, Ballesteros \& García, 2001); y una edad media de inicio al consumo de 13.46 años, inferior a los 13.7 años del estudio de López et al. (2001), y a la media de los adolescentes españoles recogida en ESTUDES 2012/2013, cifrada en 13.9 años (Ministerio de Sanidad, Servicios Sociales e Igualdad, 2014); mientras que otras investigaciones (Cabrera, Mendoza \& Gutiérrez, 2004; Orgaz, Segovia, López \& Tricio, 2005), muestran edades de inicio más precoces que las halladas en nuestros futbolistas.

Los últimos datos obtenidos de la Encuesta sobre uso de drogas en enseñanzas secundarias en España (ESTUDES 2014/2015) (Ministerio de Sanidad, Servicios Sociales e Igualdad, 2016), muestran que la prevalencia del consumo actual de bebidas alcohólicas en los adolescentes españoles de 14 a 18 años es de $68.2 \%$, dato ligeramente superior al $56.2 \%$ obtenido en los futbolistas cadetes de nuestra investigación. No obstante, el patrón de consumo observado pone de manifiesto un mayor consumo ocasional de alcohol entre nuestros deportistas (24\%), siendo un valor muy superior a los hallados en otros estudios realizados en nuestro país, que analizan este hábito en adolescentes que practican actividad física y deportiva (Lázaro, 2011; López, et al., 2016; RuizJuan \& Ruiz-Risueño, 2010).

Estos datos son preocupantes no solo por la elevada proporción de bebedores habituales encontrada, sino también porque aproximadamente un tercio de los futbolistas cadetes lleva a cabo un consumo de riesgo o abusivo, caracterizado por episodios de embriaguez y el consumo de tres o más vasos seguidos, observándose proporciones superiores a las que se presentan en la población adolescente española, mostradas tanto en ESTUDES 2014/2015 (Ministerio de Sanidad, Servicios Sociales e Igualdad, 2016), como en el estudio Health Behaviour in School-aged Children(HBSC)-2014(Moreno, et al., 2016) realizado en escolares españoles de 11 a 18 años, si bien en ambos casos el período de estudio de las borracheras fue de 30 días. Estos datos contrastan con los encontrados por Meseguer (2008) que observó que los estudiantes de la ESO que realizaban actividad física con regularidad, tenían una menor prevalencia de episodios de embriaguez. Sin embargo, Castañeda y Romero (2014) y Yusko, Buckman, White y Pandina (2008) señalaron que los estudiantes universitarios deportistas de sus respectivos trabajos consumían alcohol de forma ocasional, pero cuando lo hacían presentaban una ingesta de alcohol elevada, aspecto que se pone de manifiesto en nuestro trabajo al encontrarse asociación entre todas las variables que definen el consumo de riesgo y el consumo de alcohol en la actualidad. Resulta asimismo llamativo que tanto el consumo actual de alcohol, como el consumo abusivo, son más frecuentes entre los futbolistas que toman otro tipo de drogas (legales e ilegales), han mantenido relaciones sexuales, $\mathrm{y}$ tienen conductas violentas. Si bien otros autores no estudian la asociación entre el consumo de alcohol y otras conductas de riesgo para la salud en deportistas, sí ponen de manifiesto el consumo de diversas sustancias y la adopción de conductas violentas entre los mismos (Denham, 2014; Garry \& Morrissey, 2000; Meseguer, 2008; Moore \& Werch, 2005; Veliz, et al., 2015; Veliz \& McCabe, 2015; Yusko, et al., 2008), siendo incluso estas más frecuentes en los atletas que compiten en deportes colectivos (Lorente, et al., 2004; Vaquero-Cristóbal, et al., 2012).

Diversos estudios han analizado la relación entre distintos aspectos que caracterizan la práctica deportiva y las conductas de salud. En este sentido, Castillo et al. (2007) y Rockafellow y Saules (2006), plantean que la motivación para su realización pudiera ser un factor que se asocie al consumo de alcohol. Así, los motivos no inherentes a la práctica deportiva o extrínsecos, se asocian a una mayor prevalencia de conductas nocivas, especialmente entre los deportistas de equipo. En nuestro estudio a pesar de que el $81.2 \%$ de los futbolistas declaró como motivos principales para jugar al fútbol, aspectos extrínsecos al mismo, como el gusto, la diversión y el encuentro con amigos, no se observó asociación de esta variable con el consumo actual de alcohol, ni con las variables que definen el consumo de riesgo.

En la misma línea, algunos estudios que analizan el consumo de alcohol en deportistas de diferentes modalidades han encontrado diferencias según el tipo de deporte practicado, y la intensidad de la práctica, siendo más frecuente el consumo abusivo y de riesgo entre aquellos que practican deportes competitivos y de contacto (Denham, 2014; Veliz, et al., 2015), deportes dominados por hombres (Moore \&Werch, 2005), y los que lo practican con mayor intensidad (Ruiz-Juan \& RuizRisueño, 2010); mientras que para Lorente et al. (2004) el consumo abusivo es menos frecuente entre los que participan en competiciones nacionales o internacionales. En nuestro estudio, se observó que tanto la proporción de futbolistas bebedores, como la de deportistas que consumían tres omás vasos seguidos en losúltimos 12 meses («binge drinking») fueron más frecuentes entre los futbolistas que jugaban en la división superior de preferente, que entre los que lo hacían en primera, lo que podría estar relacionado con el mayor nivel de competitividad presente en la máxima categoría.

A pesar de la asociación que se pudiera establecer entre estos factores relacionados con la práctica deportiva y el consumo de alcohol, para autores como Meseguer (2008), los adolescentes deportistas no son ajenos a los factores personales o sociales que determinan la adopción de conductas nocivas para la salud. En este sentido es necesario recordar que los jóvenes buscan su identidad y construyen su personalidad entre el grupo de iguales, siendo muy vulnerables a las presiones del grupo, que puede influir de manera importante en las conductas y las normas de este (Balaguer, 2002; Gómez, Ruiz, García, Flores \& Barbero, 2008). En nuestro trabajo, la influencia del grupo de iguales se puso de manifiesto a través de las asociaciones observadas entre las percepciones sobre las diferentes conductas de riesgo desarrolladas por sus amigos y el propio consumo de alcohol; y las encontradas entre dichas conductas de riesgo del grupo de iguales y todas las variables que caracterizan el consumo de riesgo de bebidas alcohólicas. Esto puso de manifiesto que un entorno de amigos consumidor de tabaco y de drogas, que se emborracha y adopta conductas desafiantes y violentas fue percibido con mayor frecuencia por los futbolistas bebedores y por aquellos que desarrollaban un consumo de riesgo o bebían de manera abusiva, resultados que coinciden con los obtenidos por Paniagua, García, Castellano, Sarrallé y Redondo (2001), y por Mulassi et al. (2010). 
Algunas habilidades personales percibidas por nuestros futbolistas, como la popularidad y la capacidad de liderazgo fueron más frecuentes entre los bebedores actuales; mientras que esta última, junto con la capacidad de ser divertidos y de hacer amigos fácilmente lo fueron entre los deportistas que desarrollaban un consumo abusivo de alcohol. En esta línea Pastor, Balaguer y García-Merita (2006), destacan que una mayor percepción de la habilidad para hacer amigos íntimos, se relaciona con un mayor consumo de alcohol. Por otro lado, nuestros resultados son coherentes con lo manifestado por Espada, Pereira y GarcíaFernández (2008) y Moreno (2006), que sugieren que el consumo de alcohol se asocia en los medios de comunicación social a imágenes relacionadas con la satisfacción personal, el placer y la popularidad, favoreciendo efectos de modelado en el consumo de los adolescentes.

Asimismo, la percepción de los futbolistas de no servir para la escuela se asoció con un mayor consumo de alcohol, coincidiendo con lo descrito en estudios realizados en adolescentes de esta edad (Alves, Almeida \& Fernandes, 2017). Autores como Martínez-Lorca y AlonsoSanz (2003), destacan la importancia de este aspecto, sobre el rendimiento escolar, ya que este último ha mostrado escasa relación con el consumo de drogas, mientras que la percepción de servir para la escuela sí ha demostrado tener un efecto protector respecto a dicho consumo.

Al valorar la posible influencia de las familias de nuestros deportistas en el consumo de alcohol de sus hijos, se apreció que la percepción de un entorno familiar caracterizado por un escaso control de los mismos fue más frecuente tanto entre los que consumían alcohol, como entre los que lo hacían de manera abusiva. Las percepciones relacionadas con una escasa afectividad por parte de las familias también se asociaron al consumo de bebidas alcohólicas. Todo ello, parece indicar que el consumo y el abuso de las mismas podrían estar determinados por un modelo familiar permisivo y poco afectivo. Un estilo educativo parental poco controlador, y las relaciones familiares carentes de afecto entre padres e hijos, son importantes factores de riesgo asociados al consumo de sustancias por parte de sus hijos, tal como se ha puesto de manifiesto en otros trabajos (Dever, Schulenberg, Dworkin, O’Malley, Kloska \& Bachman, 2012).

Aunque ambos tipos de consumo son más frecuentes entre los futbolistas que juegan en preferente, nuestros resultados ponen de manifiesto, sobre todo, que estos están asociados a factores externos a la práctica deportiva. Entre los mismos destacan la adopción de otras conductas de riesgo para la salud, la percepción de liderazgo y popularidad entre los iguales y la percepción de no servir para la escuela; la percepción de un estilo educativo parental poco controlador y carente de afecto; y un entorno de iguales que consume drogas legales e ilegales, y que desarrolla conductas violentas y desafiantes. La práctica de fútbol por parte de los adolescentes de nuestro estudio no parece discriminar su estilo de vida respecto a los de otros chicos de su edad. Todo ello, unido a la elevada participación de niños y adolescentes en equipos de fútbol base, sugiere la idoneidad de este contexto para promover estilos de vida saludable. En consecuencia, se plantea la necesidad de diseñar estrategias de intervención para la prevención del consumo de bebidas alcohólicas a través de los clubes de fútbol base, teniendo en cuenta los factores individuales, sociales y familiares que determinan el mismo, donde los técnicos deportivos podrían ser una pieza clave de la formación integral del deportista.

Este estudio presenta varias limitaciones que deben ser consideradas. En primer lugar, dado el diseño del estudio, al ser una investigación desarrollada únicamente en futbolistas cadetes, no podemos determinar a pesar de la elevada prevalencia de consumo del alcohol y la elevada proporción de consumidores abusivos hallada entre los mismos, el efecto que esta práctica deportiva ejerce en la adopción de dichas conductas. En segundo lugar, las diferencias metodológicas a la hora de valorar el consumo de alcohol y el consumo de riesgo de bebidas alcohólicas, dificultan en ocasiones la comparación de resultados entre los diferentes estudios.

Como conclusión podemos afirmar que la mayoría de los futbolistas cadetes de la provincia de Las Palmas consume bebidas alcohólicas, desarrollando un alto nivel de consumo de riesgo.

\section{Agradecimientos}

Los autores de este trabajo quieren agradecer la colaboración de los cuerpos técnicos, directivos y jugadores de cada uno de los equipos de fútbol participante de este estudio.

\section{Referencias}

Alfonso, J.P., Huedo-Medina, T.B., \& Espada, J.P. (2009). Factores de riesgo predictores del patrón de consumo de drogas durante la adolescencia. Anales de Psicología, 25(2), 330-338.

Alves, D.M., Almeida, L.M., \& Fernandes H.M. (2017). Estilos de vida e autoconceito: um estudo comparativo em adolescentes. $R e$ vista Iberoamericana de Psicología del Ejercicio y del Deporte, 12(2), 237-247.

Balaguer, I. (2002). Estilos de vida en la adolescencia. Valencia: Promolibro.

Cabrera, J.L., Mendoza, M.C., \& Gutiérrez, F. (2004). Consumo de alcohol en adolescentes de tres municipios de Lanzarote. Semergen, 30(5), 210-217.

Castañeda, C., \& Romero, S. (2014). Alimentación y consumo de sustancias (alcohol, tabaco y drogas) del alumnado universitario. Análisis en función del género y la práctica de actividad físico-deportiva. Cultura, Ciencia, Deporte, 9(26), 95-105.

Castillo, I., Balaguer, I., \& García-Merita, M. (2007). Efecto de la práctica de actividad física y de la participación deportiva sobre el estilo de vida saludable en la adolescencia en función del género. Revista de Psicología del Deporte, 16(2), 201-210.

Denham, B.E. (2014). High school sports participation and substance use: differences by sport, race, and gender. Journal of Child \& Adolescent Substance Abuse, 23, 145-154. doi: 10.1080/ 1067828X.2012.750974.

Dever, B.V., Schulenberg, J.E., Dworkin, J.B., O’Malley, P.M., Kloska, D.D., \& Bachman, J.G. (2012). Predicting risk-taking with and without substance use: the effects of parental monitoring, school bonding, and sports participation. Prevention Science, 13(6), 605615. doi:10.1007/s11121-012-0288-z.

Espada, J.P., Méndez, F.X., \& Hidalgo, M.D. (2000). Consumo de alcohol en escolares: descenso de la edad de inicio y cambios en los patrones de ingesta. Adicciones, 12(1), 57-64.

Espada, J.P., Pereira, J.R., \& García-Fernández, J.M. (2008). Influencia de los modelos sociales en el consumo de alcohol en los adolescentes. Psicothema, 20(4), 531-537.

García-Moreno, L.M., Expósito, J., Sanhueza, C., \& Angulo, M.T. (2008). Actividad prefrontal y alcoholismo de fin de semana en jóvenes. Adicciones, 20(3), 271-280.

Garry, J.P., \& Morrissey, S.L. (2000). Team sports participation and risk-taking behaviors among a biracial middle school population. Clinical Journal of Sport Medicine, 10, 185-190.

Gómez, M., Ruiz, F., García, F., Flores, G., \& Barbero, G. (2008). Razones que influyen en la inactividad físico-deportiva en la Educación Secundaria Post Obligatoria. Reto. Nuevas Tendencias en Educación Física, Deporte y Recreación, 14, 80-85.

Lázaro, C.J. (2011). Hábito de consumo de alcohol y su relación con la condición física saludable en adolescentes de la Región de Murcia (Tesis doctoral). Universidad de Murcia, Murcia.

Lema, L.F., Salazar, I.C., Varela, M.T., Tamayo, J.A., Rubio, A., \& Botero, A. (2009). Comportamiento y salud de los jóvenes universitarios: satisfacción con el estilo de vida. Pensamiento Psicológico, 5(12), 71-88.

López, J.R., Antolín, N., Barceló, M.V., Pérez. M., Ballesteros, A.M., \& García,A.L. (2001). Consumo de alcohol en escolares de unárea de salud. Hábitos y creencias. Atención Primaria, 27(3), 159-165. doi: 10.1016/S0212-6567(01)78790-2

López, F.J., Rodríguez, P.L., García, E., \& Pérez, J.J. (2016). Relación entre la práctica físico-deportiva y el consumo de alcohol en adolescentes escolarizados de Murcia (España). Archivos Argentinos de 
Pediatría,114(2), 101-106.http://dx.doi.org/10.5546/aap.2016.101.

Lorente, F.O., Souville, M., Griffet, J., \& Grélot, L. (2004). Participation in sports and alcohol consumption among French adolescents. Addictive Behaviors, 29, 941-946. doi:10.1016/ j.addbeh.2004.02.039.

Martínez-Lorca, M., \& Alonso-Sanz, C. (2003). Búsqueda de sensaciones, autoconcepto, asertividad y consumo de drogas ¿Existe relación? Adicciones, 15(2), 145-158.

Meseguer, C. (2008). Práctica deportiva, niveles de actividad física habitual y consumo de alcohol en adolescentes escolarizados de la Región de Murcia (Tesis doctoral). Universidad de Murcia, Murcia.

Ministerio de Sanidad, Política Social e Igualdad(2012). Encuesta estatal sobre uso de drogas en estudiantes de enseñanzas secundarias. ESTUDES 2010. España. Madrid: Plan Nacional sobre Drogas.

Ministerio de Sanidad, Servicios Sociales e Igualdad (2014). Encuesta estatal sobre uso de drogas en estudiantes de enseñanza secundaria de 14 a 18 años. ESTUDES 2012/2013. España. Recuperado de https://www.msssi.gob.es/gabinete/notasPrensa.do?id=3218.

Ministerio de Sanidad, Servicios Sociales e Igualdad (2016). Encuesta estatal sobre uso de drogas en estudiantes de enseñanza secundaria en España. ESTUDES 2014/2015. España. Madrid: Plan Nacional sobre Drogas.

Moore, M.J., \& Werch, C.E. (2005). Sport and physical activity participation and substance use among adolescents. Journal of Adolescent Health, 26, 486-493. doi: 10.1016/ j.jadohealth.2004.02.031.

Moreno, J. (2006). Valores, actitudes hacia el alcohol y consume en adolescentes varones. Límite. Revista de filosofía y psicología, 1(13), 195-211.

Moreno, C., Ramos, P., Rivera, F., Jiménez-Iglesias, A., García-Moya. I., Sánchez-Queija, I . ..., \& Granado, M.C. (2012). Las conductas relacionas con la salud y el desarrollo de los adolescentes españoles. Resultados del estudio HBSC, 2010 con chicos y chicas españoles de 11 a 18 años. Madrid: Ministerio de Sanidad, Servicios Sociales e Igualdad.

Moreno, C., Ramos, P., Rivera, F., Jiménez-Iglesias, A., García-Moya. I., Sánchez-Queija, I. ..., \& Morgan, A. (2016). Los adolescentes españoles: estilos de vida, salud, ajuste psicológico y relaciones en sus contextos de desarrollo. Resultados del Estudio HBSC-2014 en España. Madrid: Ministerio de Sanidad, Servicios Sociales e Igualdad.

Motos, P., Cortés, M.T., Giménez, J.A. \& Cadaveira, F. (2015). Predictores del consumo semanal de alcohol y sus consecuencias asociadas en universitarios consumidores intensivos de alcohol. Adicciones, 27(2), 119-131.

Moure-Rodríguez, L., Doallo, S., Juan-Salvadores, P., Corral, M., Cadaveira, F. \& Caamaño-Isorna, F. (2016). Consumo intensivo de alcohol y cannabis, y prácticas sexuales de riesgo en estudiantes universitarios. Gaceta Sanitaria, 30(6), 438-443. http://dx.doi.org/ 10.1016/j.gaceta.2016.03.007.

Mulassi, A.H, Hadid, C., Borraci, R.A., Labruna, M.C., Picarel, A.E., Robilotte, A.N. ..., \& Masoli, O. (2010). Hábitos de alimentación, actividad física, tabaquismo, y consumo de alcohol en adolescentes escolarizados de la provincia y el conurbano bonaerenses. Archivos Argentinos de Pediatría, 108(1), 45-54.

OMS (2008). Glosario de términos de alcohol y drogas. Madrid: Ministerio de Sanidad y Consumo.

Orgaz, M.P., Segovia, M., López, F., \& Tricio. M.A. (2005). Consumo de alcohol en escolares toledanos: motivos y alternativas. Atención Primaria, 36(6), 297-305. doi: 10.1157/13079862.

Paniagua, H., García, S., Castellano, G., Sarrallé, R., \& Redondo, C. (2001). Consumo de tabaco, alcohol y drogas no legales entre adolescentes y relación con los hábitos de vida y el entorno. Anales españoles de pediatría, 55(2), 121-128.

Pastor, Y., Balaguer, I., \& García-Merita, M. (2006). Relaciones entre en el autoconcepto y el estilo de vida saludable en la adolescencia media: un modelo exploratorio. Psicothema, 18(1), 18-24.

Peck, S.C., Vida, M., \& Eccles, J.S. (2008). Adolescent pathways to adulthood drinking: sport activity involvement is not necessarily risky or protective. Addiction, 103(suppl. 1), 69-83. doi:10.1111/ j.1360-0443.2008.02177.x.

Poortinga, W. (2007). Associations of physical activity with smoking and alcohol consumption: a sport or occupation effect? Preventive Medicine, 45(1), 66-70. doi: 10.1016/j.ypmed.2007.04.013.

Rockafellow, B.D., \& Saules, K.K. (2006). Substance use by college students: the role of intrinsic versus extrinsic motivation for athletic involvement. Psychology of Addictive Behaviors, 20(3), 279-287. doi: 10.1037/0893-164X.20.3.279.

Ruiz-Juan, F., \& Ruiz-Risueño, J.R. (2010). Ingesta de alcohol y práctica de actividad físico-deportiva en jóvenes. Revista Internacional de Medicina y Ciencias de la Actividad Física yel Deporte, 10(38), 302-322.

Sañudo, B., de Hoyo, M., \& Corral, J.A. (2009). Estilo de vida de adolescentes sevillanos: actividad física y consumo de alcohol. Tándem: Didáctica de la Educación Física, 31, 62-70.

VanKim, N.A., Nelson, M., Ehlinger, E., Lust, K. \& Story, M. (2010). Understanding young adult physical activity, alcohol and tobacco use in community colleges and 4-year post-secondary institutions: A cross-sectional analysis of epidemiological surveillance data. BMC Public Health, 10(208). doi: 10.1186/1471-2458-10-208.

Vaquero-Cristóbal, R., Isorna, M., \& Ruiz, C. (2012). Revisión sobre la situación actual del consumo de alcohol y práctica físico-deportiva. Journal of Sport and Health Research, 4(3): 269-288.

Veliz, P., \& McCabe, S.E. (2015). Examining potential substance use disorders among former interscholastic athletes. Substance Abuse, 36(4), 400-406. doi: 10.1080/08897077.2014.988324.

Veliz, P.T., Boyd, C.J., \& McCabe, S.E (2015). Competitive sport involvement and substance use among adolescents: a nationwide study. Substance Use Misuse, 50(2), 156-165. doi:10.3109/ 10826084.2014.962049.

Yusko, D.A., Buckman, J.F., White. H.R., \& Pandina, R.J. (2008). Alcohol, tobacco, illicit drugs, and performance enhancers: a comparison of use by college student athletes and nonathletes. Journal of American College Health, 57(3), 281-289. doi:10.1016/ j.addbeh.2008.07.010.

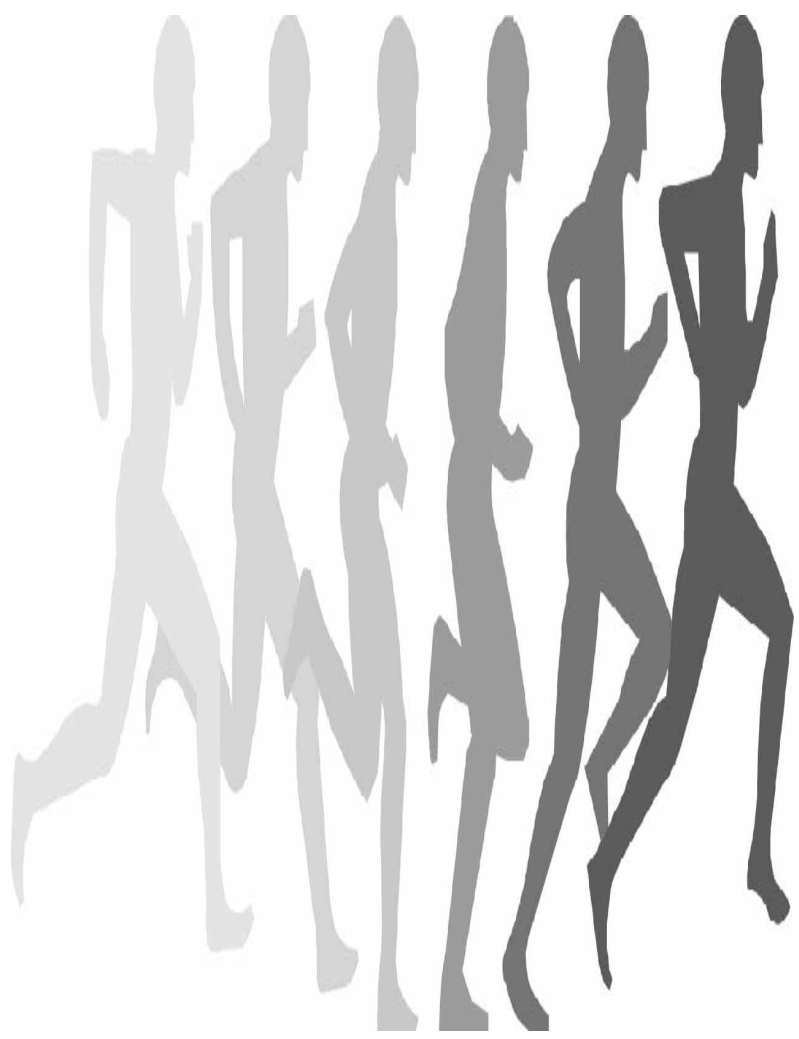

